\title{
Raman Lidar Profiles-Temperature (RLPROFTEMP) Value-Added Product
}

RK Newsom

C Sivaraman

SA McFarlane

October 2012

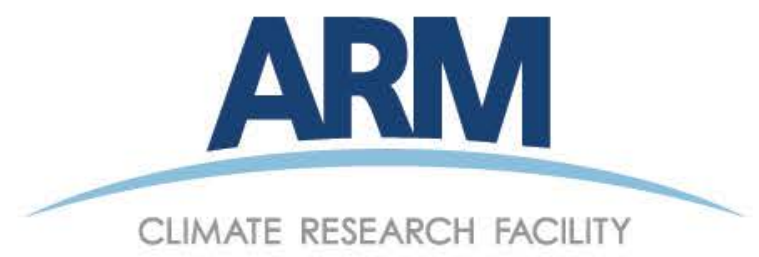




\section{DISCLAIMER}

This report was prepared as an account of work sponsored by the U.S. Government. Neither the United States nor any agency thereof, nor any of their employees, makes any warranty, express or implied, or assumes any legal liability or responsibility for the accuracy, completeness, or usefulness of any information, apparatus, product, or process disclosed, or represents that its use would not infringe privately owned rights. Reference herein to any specific commercial product, process, or service by trade name, trademark, manufacturer, or otherwise, does not necessarily constitute or imply its endorsement, recommendation, or favoring by the U.S. Government or any agency thereof. The views and opinions of authors expressed herein do not necessarily state or reflect those of the U.S. Government or any agency thereof. 


\section{Raman Lidar Profiles-Temperature (RLPROFTEMP) Value-Added Product}

RK Newsom

C Sivaraman

SA McFarlane

October 2012

Work supported by the U.S. Department of Energy,

Office of Science, Office of Biological and Environmental Research 


\section{Contents}

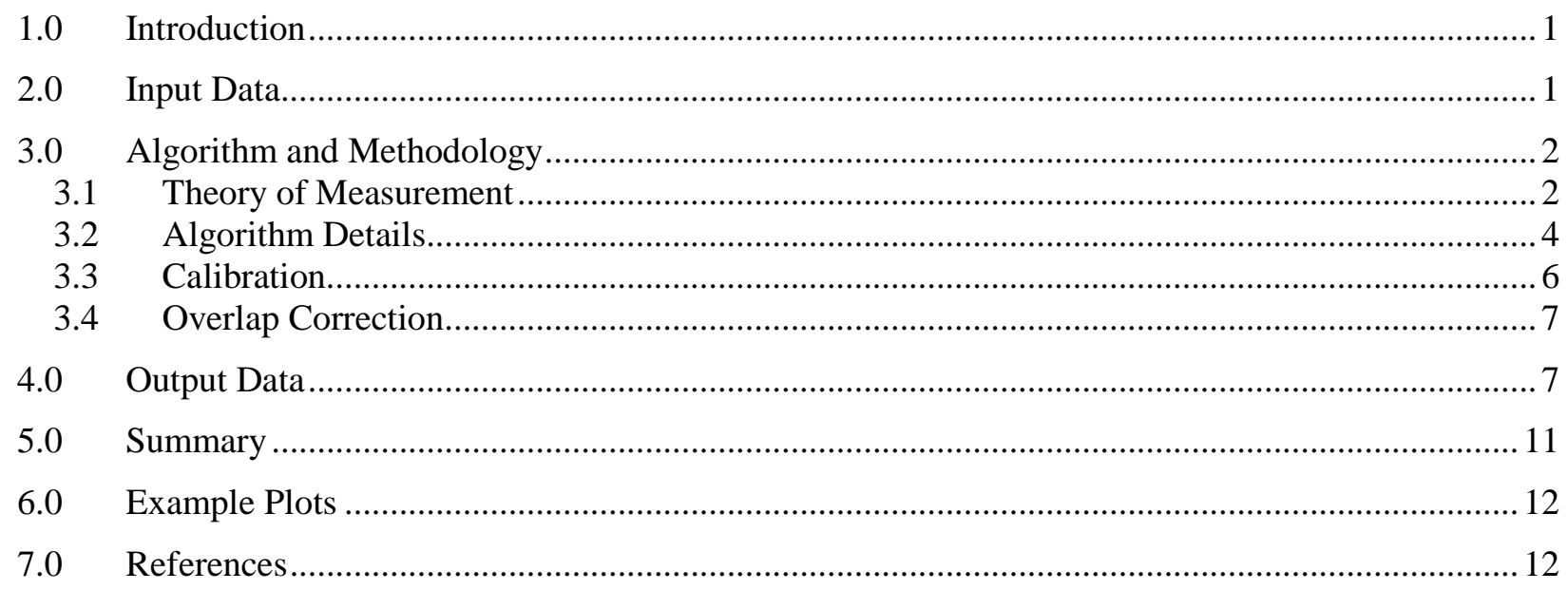

\section{Figures}

Figure 1. Interference filter transmittances (solid dar Radlach k) for the low-J (RR1) and high-J

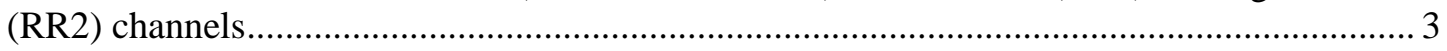

Figure 2. Flow diagram of the RL temperature VAP process......................................................... 5

Figure 3. Calibration coefficients for the RL temperature measurements are determined from a linear regression between the natural $\log$ of the RR signal ratio, $\ln Q$, and the

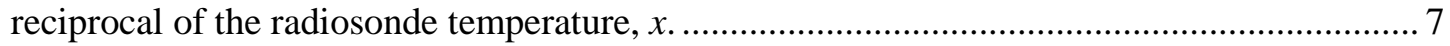

Figure 4. Sample output from the Raman lidar temperature VAP covering four selected months ........ 12 


\subsection{Introduction}

Temperature is one of the most fundamental atmospheric state variables. The temperature profile in the free troposphere and boundary layer determines stability and is critical to understanding many meteorological processes, e.g., convection or the propagation of gravity waves.

There are currently a number of Atmospheric Radiation Measurement (ARM) Climate Research Facility instruments capable of profiling temperature on a routine basis. These include the balloon-borne sounding system (SONDE), the atmospheric emitted radiance interferometer (AERI), the microwave radiometer profiler (MWRP), and the Raman lidar (RL). Each of these instruments have strengths and weaknesses. Radiosondes are capable of providing high quality measurements with good vertical resolution. However, operational and cost constraints limit the frequency with which these instruments can be launched, resulting in temporally under-sampled observations, particularly in the lower troposphere. The passive remote sensors (AERI and MWRP), which rely on statistical or physical retrieval techniques to estimate temperature, can provide measurements with much better temporal resolution, but with coarser vertical resolution and more limited height coverage (Feltz et al. 2003, Feltz et al. 2005, Loehnert et al. 2009). The RL is currently the only ARM active remote sensing instrument capable of profiling temperature through most of the free troposphere. By contrast to the passive techniques, the RL provides a more direct measurement of temperature with potentially better accuracy in the free troposphere.

The purpose of this document is to describe the Raman Lidar Profiles-Temperature (RLPROFTEMP) value-added product (VAP) and the procedures used to derive atmospheric temperature profiles from the raw RL measurements. Sections 2 and 4 describe the input and output variables, respectively. Section 3 discusses the theory behind the measurement and the details of the algorithm, including calibration and overlap correction. Validation of the RL temperature data and additional details about the algorithm are also described by Newsom et al. (2012).

\subsection{Input Data}

All required input datastreams and variables are listed below.

1. Datastream: rlprofmerge1turn

a. Data level: c0

b. Site/Facility, time period

i. SGP/C1, 20051027 to present

ii. TWP/C3, 20101215 to present

c. Variables

i. base_time

ii. time_offset

iii. shots_summed

iv. pulse_energy 

v. filter
vi. cbh
vii. t1_counts_high
viii.t2_counts_high
ix. lat
$\mathrm{x}$. lon
xi. alt

2. Datastream: sondewnpn

a. Data level: b1

b. Site/Facility, time period

i. SGP/C1, 20051027 to present

ii. TWP/C3, 20101215 to present

c. Variables
i. base_time
ii. time_offset
iii. pres
iv. tdry
v. frh
vi. alt

\subsection{Algorithm and Methodology}

\subsection{Theory of Measurement}

The RL measures atmospheric temperature using the ratio of two signals from the lidar's rotational Raman (RR) channels. The RR channels sense Raman-shifted backscatter arising from rotational energy state transitions in atmospheric $\mathrm{N}_{2}$ and $\mathrm{O}_{2}$ molecules due to excitation at the laser wavelength of $354.7 \mathrm{~nm}$ (Di Girolamo et al. 2004, Behrendt et al. 2004, Radlach et al. 2008). These channels use very narrowbandwidth interference filters to measure the energy content in two different bands in the anti-Stokes branch of the rotational spectrum, as illustrated in Figure 1. The measurements correspond to low- and high-J state transitions, where $\mathrm{J}$ denotes the rotational quantum number of the molecule. 


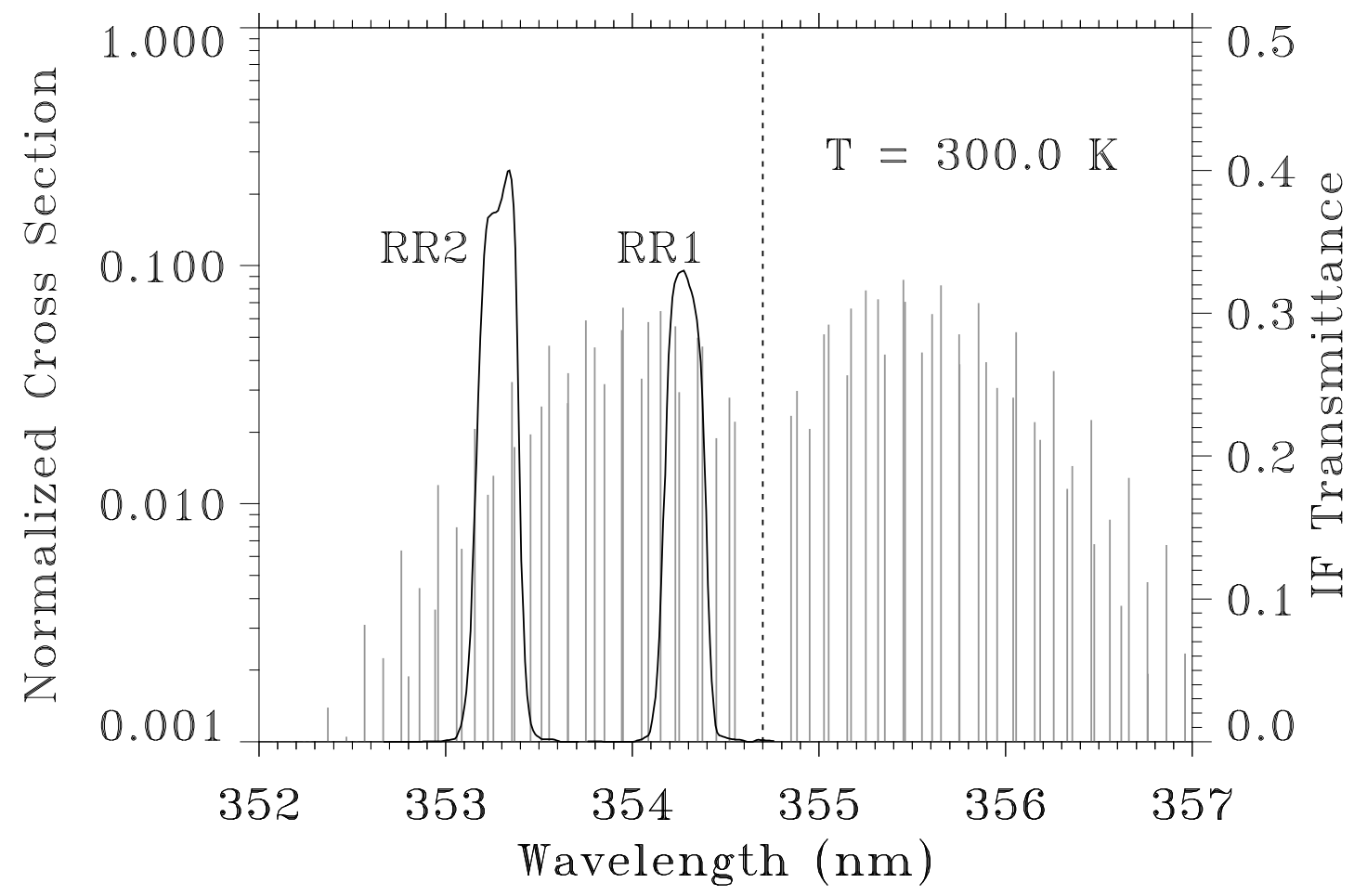

Figure 1. Interference filter transmittances (solid dark) for the low-J (RR1) and high-J (RR2) channels. The normalized intensities of the RR lines for air at $300 \mathrm{~K}$ are shown as the solid gray vertical lines. The transmit wavelength is indicated by the vertical dotted line. The center wavelengths of the two RR channels are 353.3 and $354.3 \mathrm{~nm}$, and both bands have a fullwidth-at-half max of $0.2 \mathrm{~nm}$.

The ratio of the RR signals is largely dependent on the temperature of the scattering volume and the effect of incomplete overlap between the outgoing beam and the field-of-view of the receiver. Differential transmission effects are negligible because the wavelength difference between the two RR channels is small. The height dependence of the ratio of the RR signals, $Q$, is well approximated by

$$
Q(z) \equiv \frac{S_{1}(z)}{S_{2}(z)}=O(z) \exp \left(a+b / T^{\prime}(z)\right)
$$

where $S_{1}$ and $S_{2}$ are the background-subtracted RR signals at $354.3 \mathrm{~nm}$ and $353.3 \mathrm{~nm}$, respectively; $O$ is the overlap function; $\mathrm{z}$ is the height above the lidar; $a$ and $b$ are calibration coefficients; and $T^{\prime}$ is the normalized temperature (Behrendt and Reichardt 2000). The temperature is normalized according to

$$
T^{\prime}=T / 300 K
$$

The scale factor of $300 \mathrm{~K}$ is used simply to make the calibration constant $b$ non-dimensional and of the same order of magnitude as $a$.

Computing the temperature from equation (1) first requires determination of the two calibration coefficients and the overlap function. Once these quantities are established the temperature is computed by simply solving equation (1) for $T$ : 


$$
T=\frac{(300 K) b}{\ln (Q / O)-a}
$$

The uncertainty in $T$ is obtained using standard error propagation methods. The result is given by

$$
\left(\frac{\delta T}{T}\right)^{2}=T^{\prime 2}\left(\frac{\delta Q}{b Q}\right)^{2}+T^{\prime 2}\left(\frac{\delta a}{b}\right)^{2}+\left(\frac{\delta b}{b}\right)^{2}
$$

Equation (4) accounts for uncertainties in signal ratio, $Q$, and the two calibration coefficients, $a$ and $b$, but does not include any contribution from the uncertainties in the overlap function. Estimating the uncertainties in the overlap function is inherently difficult, and so no attempt was made to include it in the overall error estimate of $T$. The details concerning the estimation of the calibration coefficients and the overlap function are described in the following sections.

\subsection{Algorithm Details}

The structure of the RLPROFTEMP VAP process is modeled after the existing Raman lidar VAPs. Most of the signal preprocessing steps are similar, and many of the same subroutines are used. The main routine is called by specifying the date, the site and facility designations, the time averaging interval, and the paths to input data. As with the other RL VAPs, 72 hours (three days) are processed at a time. This includes the date specified in the call and the two days to either side of that date. The output is written to a netCDF file, with a format that is compliant with current ARM standards.

The basic processing steps are illustrated in Figure 2 and outlined below. In the following, $S_{1}$ and $S_{2}$ refer to the two RR channels at 354.3 and $353.3 \mathrm{~nm}$, respectively. 


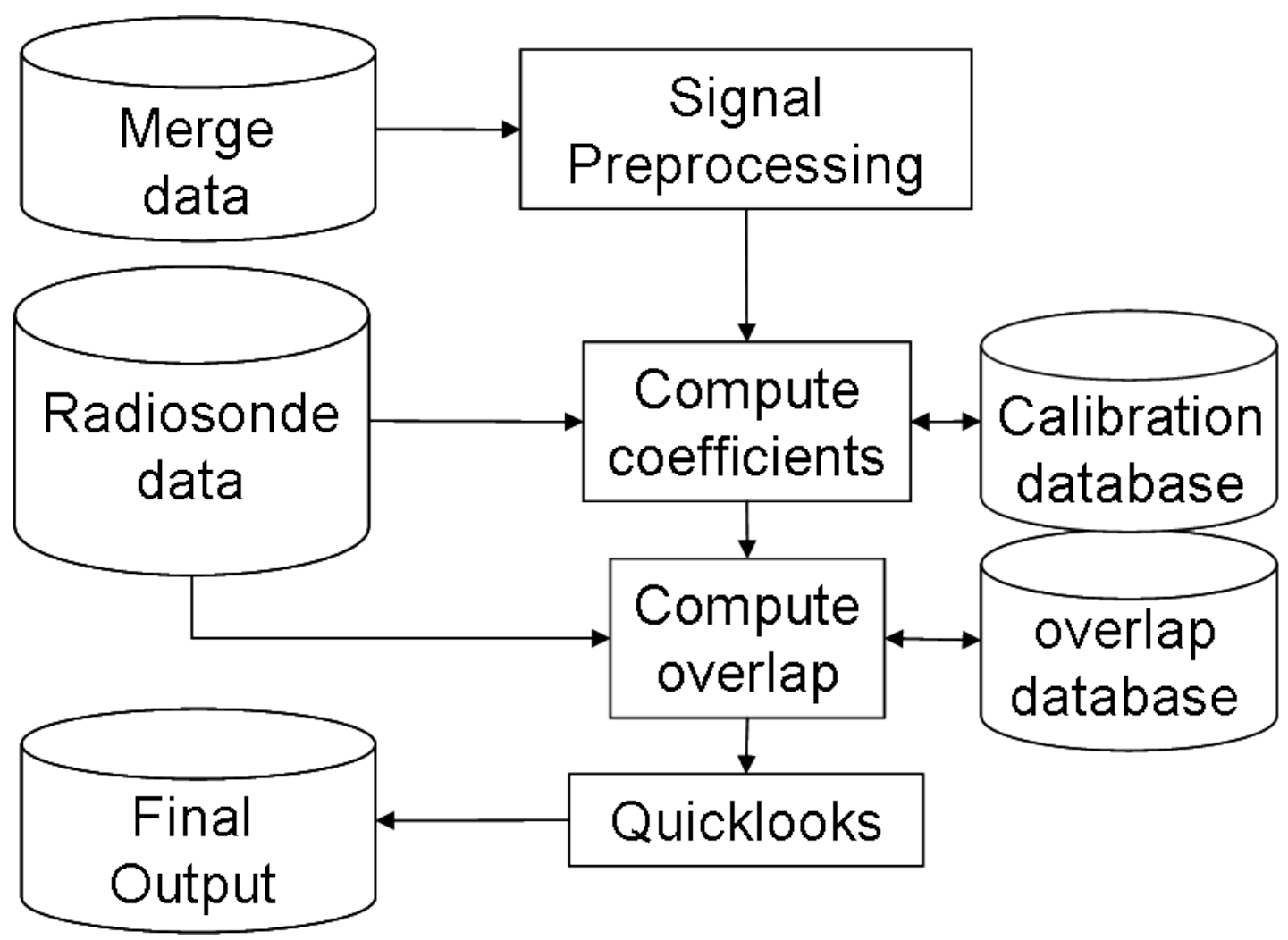

Figure 2. Flow diagram of the RL temperature VAP process.

1. Lidar signal preprocessing:

a. Read in 3 days of raw $S_{1}$ and $S_{2}$ data from the merge VAP (rlprofmerge1turn).

b. Average $S_{1}$ and $S_{2}$ over prescribed time and height bins and compute the corresponding errors, $\delta S_{1}$ and $\delta S_{2}$, assuming the signals are dominated by shot noise. The time and height resolution of the raw data is $10 \mathrm{sec}$ and $7.5 \mathrm{~m}$, respectively. The averaging time interval is prescribed through the call to the main routine, while the averaging height bin is prescribed in a user-editable configuration file.

c. Subtract the background signal levels from $S_{1}$ and $S_{2}$.

2. Read in radiosonde temperature data $T_{\text {sonde }}$ and interpolate to the lidar height grid. All available radiosondes during the 72-hour processing period are read in. The default is to use b1 level sondewnpn data.

3. Compute the ratio $Q(z)=S_{1} / S_{2}$ and the corresponding error, $\delta Q$.

4. Determine the calibration coefficients, a and b, using $Q$ and $T_{\text {sonde }}$.

5. Determine the overlap function, $O(z)$, using $Q$ and $T_{\text {sonde }}$.

6. Compute RL temperatures and write the results to a netCDF file.

7. Generate quicklook plots. 


\subsection{Calibration}

As alluded to in the outline, both calibration and overlap correction are performed using radiosonde temperature data, which are regarded as truth. The straight-line fit is obtained through samples for which $5 \mathrm{~km}<\mathrm{z}<15 \mathrm{~km}$ and $200 \mathrm{~K}<T_{\text {sonde }}<320 \mathrm{~K}$. In the region above $4 \mathrm{~km}$ above ground level, the overlap function is essentially unity ${ }^{1}$ so that equation (1) can be rewritten as

$$
\ln Q=a+b x
$$

where $x=1 / T_{\text {sonde }}$ and $T_{\text {sonde }}$ is the radiosonde temperature.

The calibration coefficients, $a$ and $b$, are determined from a linear regression between $\ln Q$, and the reciprocal of the radiosonde temperature, $x$, as illustrated in Figure 3. Only lidar measurements acquired at the time of the radiosonde launch are used in the regression analysis. No attempt is made to account for the horizontal drift of the radiosonde or the time difference between samples in the radiosonde profile. Thus, we assume that the radiosonde profile is representative of a single vertical profile at the time of launch.

Uncertainties in the calibration coefficients are also estimated as part of the regression analysis. This is done using standard error propagation techniques, in which the error in $\mathrm{Q}$ is given in terms of the errors in the individual signals:

$$
\left(\frac{\delta Q}{Q}\right)^{2}=\left(\frac{\delta S_{1}}{S_{1}}\right)^{2}+\left(\frac{\delta S_{2}}{S_{2}}\right)^{2} .
$$

The errors in the individual signals are assumed to be dominated by shot noise and thus obey Poisson distributions.

The calibration procedure first computes the so-called "background" calibration coefficients by applying the regression technique using all available radiosonde profiles over the 3-day period. If the resulting fit passes a quality assurance (QA) test ${ }^{2}$, then the calibration coefficients are saved to a database file and used as background values in the determination of time-varying coefficients. The time-varying coefficients are computed by applying the regression technique to individual radiosonde profiles while imposing the background values as a constraint. This helps to suppress large deviations from the background. If the fit for a particular sounding fails the QA test, then the background calibration is used for that time period. If the background calibration fails the QA test, then the algorithm searches the database file for calibrations closet to the current date. In this case, no attempt is made to compute timevarying calibrations.

\footnotetext{
${ }^{1}$ This applies to the narrow-field-of-view (NFOV) channels. For the wide-FOV channels complete overlap occurs for heights above $800 \mathrm{~m}$. The RR channels are only implemented in the NFOV branch of the RL receiver optics.

${ }^{2}$ The QA test uses thresholds for the maximum chi-squared and minimum linear correlation.
} 


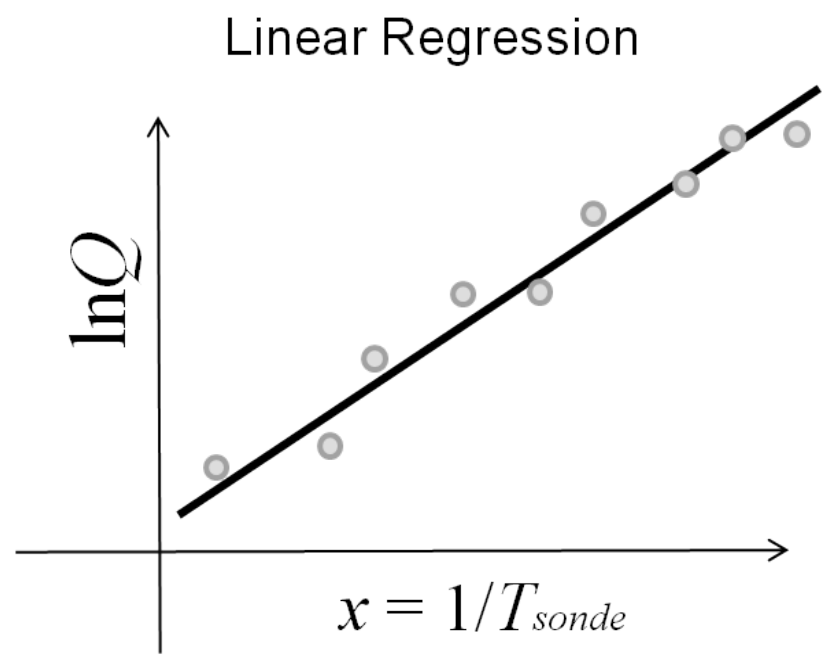

Figure 3. Calibration coefficients for the RL temperature measurements are determined from a linear regression between the natural $\log$ of the $\mathrm{RR}$ signal ratio, $\ln Q$, and the reciprocal of the radiosonde temperature, $x$.

\subsection{Overlap Correction}

Once the calibration coefficients have been determined, the overlap function is estimated using equation (1),

$$
O(z)=\left\langle Q(z, t) \exp \left(-a-b / T_{\text {sonde }}(z, t)\right)\right\rangle_{t}
$$

where \langle\rangle$_{t}$ denotes the average over all radiosonde times, $t$, at a specific height, $z$. As with calibration, the procedure for estimating the overlap function makes use of a database file that maintains a record of overlap functions from other days. If the overlap function derived from equation (4) fails the QA test, then the algorithm searches the database file for overlap functions closest in time to the current day. In contrast to the calibration procedure, no attempt is made to determine a time-varying overlap function. Instead a static overlap function is determined using all of the available radiosonde profiles during the three-day processing period.

\subsection{Output Data}

The RLPROFTEMP VAP datastream name is rlproftemp1news, and the data level is "c0" (as is the case for the other intermediate-level RL VAPs). All output variables are listed below.

netcdf twp10rlproftemp1newsC3.c0.20110106.000000 \{

dimensions:

time = UNLIMITED ; // (144 currently)

height $=200$; 
variables:

int base_time ;

base_time:string = "6-Jan-2011,00:00:00 GMT" ;

base_time:long_name = "Base time in Epoch" ;

base_time:units = "seconds since 1970-1-1 0:00:00 0:00" ;

double time_offset(time) ;

time_offset:long_name = "Time offset from base_time" ;

time_offset:units = "seconds since 2011-1-6 00:00:00 0:00" ;

double time(time) ;

time:long_name = "Time offset from midnight" ;

time:units = "seconds since 2011-1-6 00:00:00 0:00" ;

float height(height) ;

height:long_name = "Altitude" ;

height:units = "km" ;

float tp1(time, height) ;

tp1:long_name = "Background subtracted rotational Raman signal at 353nm" ;

tp1:units = "MHz" ;

float tp1_error(time, height) ;

tp1_error:long_name = "Error in the background subtracted rotational Raman signal at 353nm" ; tp1_error:units = "MHz" ;

float tp1_bkg(time);

tp1_bkg:long_name = "Background of the rotational Raman signal at 353nm" ;

tp1_bkg:units = "MHz" ;

float tp1_bkg_error(time) ;

tp1_bkg_error:long_name = "Error in the background of the rotational Raman signal at 353nm" ;

tp1_bkg_error:units = "MHz" ;

float tp2(time, height) ;

tp2:long_name = "Background subtracted rotational Raman signal at 354nm" ;

tp2:units = "MHz" ;

float tp2_error(time, height) ;

tp2_error:long_name = "Error in the background subtracted rotational Raman signal at 354nm" ;

tp2_error:units = "MHz" ;

float tp2_bkg(time) ;

tp2_bkg:long_name = "Background of the rotational Raman signal at 354nm" ;

tp2_bkg:units = "MHz" ;

float tp2_bkg_error(time) ;

tp2_bkg_error:long_name = "Error in the background of the rotational Raman signal at 354nm" ; tp2_bkg_error:units = "MHz" ;

float rot_raman_ratio(time, height) ;

rot_raman_ratio:long_name = "Raw rotational Raman ratio" ;

rot_raman_ratio:units = "unitless" ;

rot_raman_ratio:comment = "Uncorrected and uncalibrated T1/T2 ratio" ;

float rot_raman_ratio_error(time, height) ;

rot_raman_ratio_error:long_name = "Raw rotational ratio error" ;

rot_raman_ratio_error:units = "unitless" ; 
rot_raman_ratio_error:comment = "Estimated error (from shot noise only) in the uncorrected and uncalibrated T1/T2 ratio" ;

float rot_raman_temperature(time, height) ;

rot_raman_temperature:long_name = "Calibrated rotational Raman temperature" ;

rot_raman_temperature:units = "K" ;

rot_raman_temperature:comment = "Overlap corrected and calibrated temperature from the rotational Raman channels" ;

float rot_raman_temperature_error(time, height) ;

rot_raman_temperature_error:long_name = "Error in calibrated rotational Raman temperature" ;

rot_raman_temperature_error:units = "K" ;

rot_raman_temperature_error:comment = "Estimated error (from shot noise only) in the overlap

corrected and calibrated te

mperature from the rotational Raman channels" ;

float a_coef(time);

a_coef:long_name = "Calibration coefficient a" ;

a_coef:units = "unitless" ;

a_coef:comment $=$ "Calibration equation is: $\ln ($ rot_raman_ratio $)=\mathrm{a}+\mathrm{b} *(300 / \mathrm{T})$, T in $\mathrm{K}^{\text {" }}$;

float a_coef_error(time) ;

a_coef_error:long_name = "Error in calibration coefficient a" ;

a_coef_error:units = "unitless" ;

a_coef_error:comment $=$ "Calibration equation is: $\ln ($ rot_raman_ratio $)=\mathrm{a}+\mathrm{b} *(300 / \mathrm{T}), \mathrm{T}$ in $\mathrm{K}^{*}$;

float b_coef(time);

b_coef:long_name = "Calibration coefficient b" ;

b_coef:units = "unitless" ;

b_coef:comment $=$ "Calibration equation is: $\ln ($ rot_raman_ratio $)=\mathrm{a}+\mathrm{b} *(300 / \mathrm{T})$, T in $\mathrm{K}$ " ;

float b_coef_error(time) ;

b_coef_error:long_name = "Error in calibration coefficient b" ;

b_coef_error:units = "unitless" ;

b_coef_error:comment = "Calibration equation is: $\ln ($ rot_raman_ratio $)=\mathrm{a}+\mathrm{b} *(300 / \mathrm{T}), \mathrm{T}$ in $\mathrm{K}^{*}$; float a_coef_scale(time) ;

a_coef_scale:long_name = "Solar background correction factor applied to the calibration coefficient a" ;

a_coef_scale:units = "unitless" ;

a_coef_scale:comment = "Solar background correction: a_coef $=$

a_coef_uncorrected*a_coef_scale" ;

float b_coef_scale(time);

b_coef_scale:long_name = "Solar background correction factor applied to the calibration coefficient b" ;

b_coef_scale:units = "unitless" ;

b_coef_scale:comment $=$ "Solar background correction: b_coef $=$

b_coef_uncorrected*b_coef_scale" ;

float sonde_temperature(time, height) ;

sonde_temperature:long_name = "Temperature from radiosondes, interpolated to this temporal

and spatial grid" ;

sonde_temperature:units = "K" ;

float sonde_pressure(time, height) ; 
sonde_pressure:long_name = "Pressure from radiosondes, interpolated to this temporal and spatial grid" ;

sonde_pressure:units = "mb" ;

float olap_function(time, height) ;

olap_function:long_name = "Overlap function" ;

olap_function:units = "unitless" ;

float olap_chisq ;

olap_chisq:long_name = "Chi-square associated with the mean overlap correction" ;

olap_chisq:units = "unitless" ;

float olap_corr ;

olap_corr:long_name = "Correlation coefficient associated with the mean overlap correction" ;

olap_corr:units = "unitless" ;

short sonde_times(time) ;

sonde_times:long_name = "Flag indicating which samples correspond to radiosonde launch times

(1)" ;

sonde_times:units = "unitless" ;

int shots_summed(time);

shots_summed:long_name = "Number of laser shots recorded" ;

shots_summed:units = "unitless" ;

float cbh(time) ;

cbh:long_name = "Median cloud base height" ;

cbh:units $=$ "km" ;

cbh:comment = "Clear sky denoted by $-1 "$;

float lat ;

lat:long_name $=$ "North latitude" ;

lat:units = "degree_N" ;

lat:valid_min $=-90 . \mathrm{f}$;

lat:valid_max $=90 . f$;

float lon ;

lon:long_name = "East longitude" ;

lon:units = "degree_E" ;

lon:valid_min $=-180 . f$;

lon:valid_max $=180 . \mathrm{f}$;

float alt ;

alt:long_name = "Altitude above mean sea level" ;

alt:units $=$ "m" ;

// global attributes:

:process_version = "v1.0" ;

:command_line = "/home/sivaraman/dev/vap/bin/rlprof_temp -a 10 -s twp -f C3 -d 20110106" ;

:dod_version = "v1.0" ;

:site_id = "twp" ;

:facility_id = "C3" ;

:software_level = "c0" ;

:qc_standards_version = "0.1" ;

:solar_background_correction = "0" ; 
:rlprof_lib_version = "\$State: $\operatorname{Exp~\$ "~;~}$

:idl_tools_lib_version = "\$State: idltools-idltools-1.30-0.sol5_10 \$" ;

:idl_version = "VAP coded in IDL (x86_64 / sunos / unix / 8.1)" ;

:missing_data $=$ " -999.0" ;

:comment_calibration = "Calibration coefficients from 60 min average" ;

:comment_olap = "Overlap correction from 60 min average " ;

:input_datastreams_description = "A string consisting of the datastream(s), datastream version(s), and datastream date (

range)." ;

:input_datastreams_num $=13$;

:input_datastreams = "twprlprofmerge1turnC3.c0.20110106.225344.cdf : \$State: vap-

rlprof_merge-3.3-0.sol5_10 \$, twpsondew

npnC3.b1.20110105.051500.cdf : ingest-sonde-10.6-0.sol5_10, twpsondewnpnC3.b1.20110105.111500.cdf : ingest-sonde-10.6-0.sol5_10, twpsond ewnpnC3.b1.20110105.171500.cdf : ingest-sonde-10.6-0.sol5_10, twpsondewnpnC3.b1.20110105.231700.cdf : ingest-sonde-10.6-0.sol5_10, twpso

ndewnpnC3.b1.20110106.054900.cdf : ingest-sonde-10.6-0.sol5_10, twpsondewnpnC3.b1.20110106.111500.cdf : ingest-sonde-10.6-0.sol5_10, twp sondewnpnC3.b1.20110106.171500.cdf : ingest-sonde-10.6-0.sol5_10, twpsondewnpnC3.b1.20110106.231500.cdf : ingest-sonde-10.6-0.sol5_10, t wpsondewnpnC3.b1.20110107.051600.cdf : ingest-sonde-10.6-0.sol5_10, twpsondewnpnC3.b1.20110107.111500.cdf : ingest-sonde-10.6-0.sol5_10, twpsondewnpnC3.b1.20110107.171500.cdf : ingest-sonde-10.6-0.sol5_10, twpsondewnpnC3.b1.20110107.231600.cdf : ingest-sonde-10.6-0.sol5_1

$0 "$;

:history = "created by sivaraman on machine pearl on Tue Sep 25 20:55:28 2012" ; \}

\subsection{Summary}

This document provided a brief description of the RLPROFTEMP VAP, as well as its input and output variables. Validation of the RL temperature data and additional details about the algorithm are described by Newsom et al. (2012). The VAP process has been designed to run autonomously with a minimum of user intervention. It is currently being used to process data from the Raman lidars at the ARM Southern Great Plains (SGP) and Tropical Western Pacific (TWP) sites. The algorithm is currently configured to generate output at temporal resolutions of 60 and 10 minutes. 


\subsection{Example Plots}

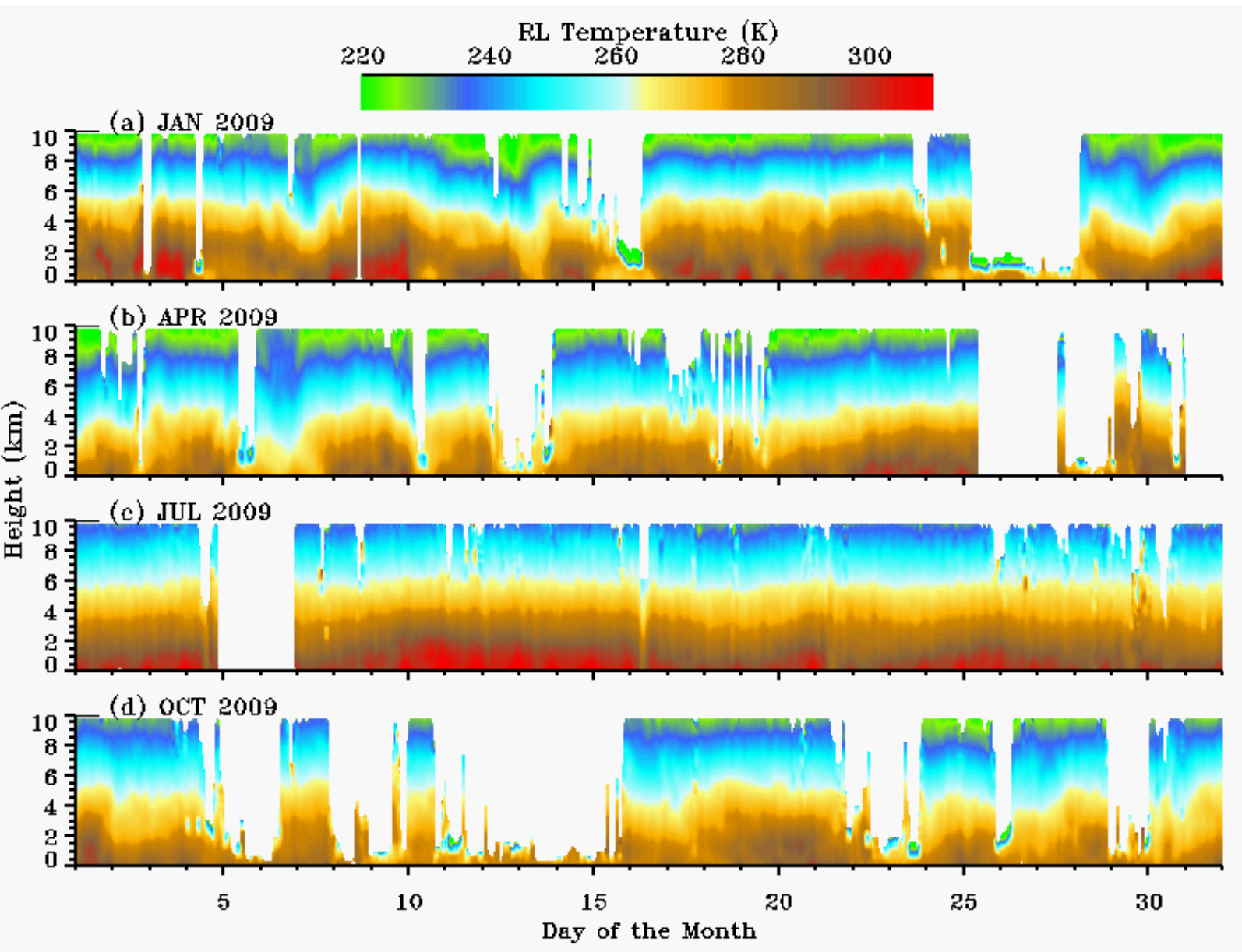

Figure 4. Sample output from the RLPROFTEMP VAP covering four selected months. Results are shown for (a) January 2009, (b) April 2009, (c) July 2009, and (d) October 2009.

\subsection{References}

Behrendt, A, and J Reichardt. 2000. “Atmospheric temperature profiling in the presence of clouds with a pure rotational Raman lidar by use of an interference-filter-based polychromator.” Applied Optics 39: 1372-1378.

Behrendt, A, T Nakamura, and T Tsuda. 2004. "Combined temperature lidar for measurements in the troposphere, stratosphere, and mesosphere.” Applied Optics 43: 2930-2939.

Di Girolamo, P, R Marchese, DN Whiteman, and B Demoz. 2004. “Rotational Raman Lidar measurements of atmospheric temperature in the UV.” Geophysical Research Letters 31, L01106, doi:10.1029/2003GL018342. 
Feltz, WF, WL Smith, HB Howell, RO Knuteson, H Woolf, and HE Revercomb. 2003. “Nearcontinuous profiling of temperature, moisture, and atmospheric stability using the Atmospheric Emitted Radiance Interferometer (AERI).” Journal of Applied Meteorology and Climatology 42, 584-597.

Feltz, WF, HB Howell, RO Knuteson, HM Woolf, DD Turner, R Mahon, TD Halther, and WL Smith. 2005. Retrieving Temperature and Moisture Profiles from AERI Radiance Observations: AERIPROF Value-Added Product Technical Description. U.S. Department of Energy. DOE/SC-ARM/TR-066.

Loehnert, U, DD Turner, and S Crewell. 2009. “Ground-based temperature and humidity profiling using spectral infrared and microwave observations. Part 1: Simulated retrieval performance in clear sky conditions.” Journal of Applied Meteorology and Climatology 48, 1017-1032, doi:10.1175/2008JAMC2060.1.

Newsom, RK, DD Turner, and JEM Goldsmith. 2012. "Long-term evaluation of temperature profiles measured by an operational Raman lidar.” Journal of Atmospheric and Oceanic Technology, submitted.

Radlach, M, A Behrendt, and V Wulfmeyer. 2008. "Scanning rotational Raman lidar at 355nm for the measurement of tropospheric temperature fields.” Atmospheric Chemistry, and Physics 8, 159-169. 


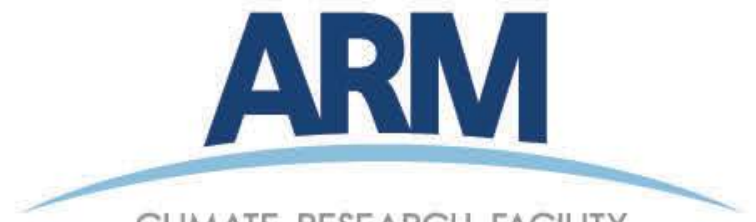

CLIMATE RESEARCH FACILITY

www.arm.gov

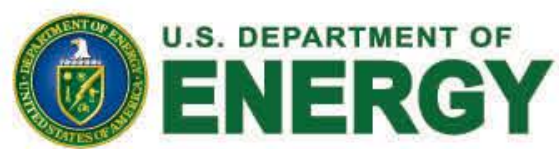

Office of Science 